\title{
Efectos de la deontología de la publicidad en la actitud profesional
}

Marta Martín Llaguno | marta.martin@ua.es

Alejandra Hernández Ruiz| alejandra.hernandez@ua.es

UNIVERSIDAD DE ALICANTE

Resumen: La ética es considerada como una competencia relevante por profesionales y académicos de la comunicación comercial. No en vano, en el contexto de la convergencia europea, la materia de deontología es obligatoria en los nuevos grados de publicidad. Sin embargo, estudios anglosajones cuestionan los efectos de los cursos de ética sobre las actitudes y los comportamientos de los publicitarios. Este trabajo mide la capacidad que ha tenido la materia de incrementar la sensibilidad hacia cuatro dimensiones del profesionalismo (la cognitivo-técnica, la social, la afectivo-emocional y la ética). Nuestro curso ha mejorado la actitud de los discentes sobre todo hacia las competencias sociales, especialmente relevantes en una profesión con un marcado carácter relacional como la publicidad.

Palabras clave: competencias profesionales, deontología publicitaria, estudiantes, género

Abstract: Ethics is considered among professionals and academics as an important competence for advertising. Not surprisingly, in the context of the European convergence, the subject of ethics is mandatory in the new degree of advertising. However, American studies on the effects of ethical training on professionals' attitudes and behaviors are not conclusive. This study assesses the impact of ethics instruction on the sensibility increase to four dimensions of professionalism (cognitive-technical, social, affective-emotional and ethical). Our course has improved students' attitudes, particularly towards social skills, especially important in a profession which emphasizes the relational competences.

Key words: Professional competences, advertising ethics, undergraduate students, gender 


\section{La importancia de la deontología publicitaria para el ejercicio profesional}

La influencia de la comunicación, a través de los medios convencionales, en la educación y en el comportamiento de los públicos receptores está fuera de toda duda (López Lita y Bernad, 2007). En particular, la publicidad tiene unos efectos sociales y culturales, más allá de sus efectos económico-comerciales primigenios que, a menudo, son ignorados (Costa, 2004). Dada la trascendencia social de este discurso, parece razonable establecer mecanismos que se encarguen de velar por una comunicación comercial veraz, legal, honesta y leal. En nuestro país, Autocontrol, organismo pionero y emblemático en cuestiones de autodisciplina publicitaria (Martín-Llaguno y Hernández, 2009), asume prioritariamente estas funciones en régimen de co-regulación ${ }^{1}$ con los poderes públicos. Según el último informe de actividad de esta asociación, en España la industria publicitaria goza de una excelente salud ética. Así, las reclamaciones en 2008 ascienden a 173, el número más bajo registrado desde el 2000. Estos resultados dan cuenta del esfuerzo que el sector profesional ha hecho desde hace años por priorizar las cuestiones deontológicas. De hecho, su sensibilidad por la ética quedaba ya explícita en el Código de Conducta Comercial en el que se expone el "compromiso por crear y sostener, en defensa del ejercicio de la ética y deontología profesional, un marco común que refleje los principios rectores generales para unas relaciones óptimas entre los distintos agentes que participan en el sector publicitario" 2 . La deontología es, por tanto, considerada como importante por los publicitarios para el ejercicio de la profesión.

\subsection{La Deontología profesional en la publicidad: una cuestión controvertida}

La relevancia que la ética adquiere en la profesión del publicitario justifica la necesidad de focalizar la atención sobre el desarrollo de esta competencia en la enseñanza universitaria.

El debate sobre la pertinencia y las características de la docencia de la ética ha irrumpido en los 90 en el campo de la comunicación comercial, donde la deontología profesional se ha vinculado con el profesionalismo y la elevación de los estándares laborales (McBride, 1989; Barney y Black, 1994; Harrison, 1990; Tucker y Stout, 1999; Bivins, 1990; Plumley y Ferragina, 1990; Wright, 1985; Gautschi III y Jones, 1998). Sin embargo, de la misma manera que sucede en otros ámbitos, en publicidad, se cuestiona la efectividad de los cursos impartidos. Los programas de ética profesional parecen influir claramente en las cogniciones, de forma que, en comunicación comercial, la obligatoriedad de la deontología en la curricula se relaciona con el conocimiento y el liderazgo ético, con la mayor consideración de las dimensiones éticas en la toma de decisiones profesionales, con la mejor capacidad para distinguir dilemas y con la disposición a no desvincular la ética personal de la profesional

\footnotetext{
${ }^{1}$ La fórmula de la co-regulación entraña que la industria es designada por el gobierno para desarrollar, aplicar o reforzar alguna norma. Ésta es la tendencia en la UE donde, con el fin de simplificar el control legal, los estamentos ejecutivos han apostado con fuerza por la aplicación y control de las normas - por ejemplo para la estrategia PAOS de la OMS (Martín-Llaguno y Hernández, 2009).

${ }^{2}$ Disponible en http://www.aeap.es/publicaciones/docs/CodigoConducta.pdf
} 
(Gale y Bunton, 2005). Sin embargo, el potencial de los cursos de deontología para modificar actitudes y comportamientos es un tema debatido desde la academia. Así, mientras algunos estudios apuntan que, tras haber asistido a un curso de deontología, los alumnos presentan cambios en el sistema de valores y un aumento de la importancia concedida a las cuestiones sociales y morales (Surlin, 1987; Gale y Bunton, 2005; Stead y Miller, 1988), otros constatan que no existe tal impacto (Martin, 1981; Borkowski y Ugras, 1992). En el caso de la comunicación comercial, aunque las habilidades críticas y analíticas y los valores de los estudiantes se ven modificados a corto plazo (Braun, 1999; Carlson y Burke, 1998; Peppas y Diskin, 2001), los cambios detectados en las actitudes (Shannon y Berl, 1997) y los comportamientos (Surlin, 1987) son escasos.

En conexión con lo anterior, hay también un extenso debate sobre la sensibilidad ética y el género. Así, en general, los estudios apuntan que las mujeres muestran actitudes más éticas que los hombres (Becker y Ulstad, 2007; Ruegger y King, 1992; Betz, 1989). Hay quien señala que la razón puede estar en que las mujeres tienden a evitar riesgos ( ianakopolos y Bernasek, 1998) e intentan eludir los sentimientos de culpa (Tibbetts, 1997; Tibbetts, 1999). En la misma línea de la influencia de los estereotipos de género, los hombres estarían condicionados porque han sido educados para ser más agresivos y competitivos (McCabe, Ingram y Dato-on, 2006).

En España, como afirma Pérez Pulido (2004), parece haber consenso en la comunidad académica sobre la relación que debe existir entre la enseñanza universitaria de la deontología y el ejercicio profesional. La ausencia de esta materia en la curricula provoca que, según esta autora, las percepciones y destrezas para conseguir conductas adecuadas dependan de la ética individual. Pese a estas opiniones, hay escasos estudios sobre el tema en nuestro país. Así, las investigaciones sobre los efectos de la enseñanza de estas materias en las actitudes de los publicistas se han realizado en un contexto cultural diferente al español, el anglosajón.

Este hecho no es de extrañar puesto que, la Ética profesional o la Deontología ni siquiera se encontraba entre las materias troncales de los antiguos planes de estudio. La materia sólo ha estado presente en algunas de las titulaciones Publicidad y de Relaciones Públicas, bien como obligatoria, bien como optativa (ver tabla 1). En la actualidad, con la convergencia europea, ha pasado a ser obligatoria.

Tabla 1. Ubicación de la Ética y la Deontología en algunos Planes de Estudios para la obtención del título de Licenciado en Publicidad y Relaciones Públicas

\begin{tabular}{|l|l|l|l|l|}
\hline Universidad & Denominación & Tipo & Curso & Créditos \\
\hline $\begin{array}{l}\text { Universitat Abat Oliva (CEU) } \\
\text { (Barcelona) }\end{array}$ & $\mathrm{x}$ & $\mathrm{X}$ & $\mathrm{x}$ & $\mathrm{x}$ \\
\hline Universitat Autónoma de Barcelona & $\mathrm{x}$ & $\mathrm{x}$ & $\mathrm{X}$ & $\mathrm{x}$ \\
\hline Universidad de Barcelona (ESRP) & $\begin{array}{l}\text { Història i Ėtica de la Publicitat i de les } \\
\text { RR.PP. a Europa i Amèrica }\end{array}$ & OP & Primero & $8 \mathrm{cr}$. \\
\hline
\end{tabular}




\begin{tabular}{|c|c|c|c|c|}
\hline Universidad & Denominación & Tipo & Curso & Créditos \\
\hline Universidad de Cádiz & $\begin{array}{l}\text { Ética y Deontología de la Publicidad y de } \\
\text { las Relaciones Públicas }\end{array}$ & OB & $\begin{array}{l}\text { Segundo } \\
\text { ciclo } \\
\text { (segundo } \\
\text { curso) }\end{array}$ & $6 \mathrm{cr}$. \\
\hline Universidad Camilo J osé Cela & Ética y Deontología Profesional & OB & Tercero & $6 \mathrm{cr}$. \\
\hline Universidad Cardenal Herrera (CEU) & Ética y Deontología & $\mathrm{OB}$ & Tercero & $\begin{array}{l}9 \mathrm{cr} . \\
\text { (anual) }\end{array}$ \\
\hline Universidad Católica de Murcia & Ética y Deontología Profesional & OB & Tercero & $7 \mathrm{cr}$. \\
\hline Universidad Complutense de Madrid & $\begin{array}{l}\text { Ética y Deontología de la Publicidad y de } \\
\text { las Relaciones Públicas }\end{array}$ & $\mathrm{OB}$ & Cuarto & $4 \mathrm{cr}$ \\
\hline Universidad Europea de Madrid & Ética y Deontología Profesional & OB & Cuarto & $6 \mathrm{cr}$. \\
\hline $\begin{array}{l}\text { Universidad Europea Miguel de } \\
\text { Cervantes }\end{array}$ & Ética y Deontología Profesional & OB & Cuarto & $6 \mathrm{cr}$. \\
\hline Universitat de Girona & $x$ & $x$ & $x$ & $x$ \\
\hline Universitat J aume I & Ética y Deontología Profesional & OB & Tercero & $4,5 \mathrm{cr}$. \\
\hline Universidad de Málaga & Deontología de la Comunicación & OP & $\begin{array}{l}\text { Segundo } \\
\text { Ciclo }\end{array}$ & $6 \mathrm{cr}$. \\
\hline $\begin{array}{l}\text { Universidad Miguel Hernández } \\
\text { (Escuela Superior de Gestión } \\
\text { Comercial y Marketing-ESIC-Valencia) }\end{array}$ & Ética de la Empresa & OP & Quinto & ¿? \\
\hline Universidad de Murcia & Ética y Deontología Profesional & OP & $\begin{array}{l}\text { Segundo } \\
\text { ciclo } \\
\text { (primer } \\
\text { curso) }\end{array}$ & $5 \mathrm{cr}$. \\
\hline Universidad de Navarra & $\begin{array}{l}\text { Deontología de la Publicidad y de las } \\
\text { RR.PP }\end{array}$ & OB & Cuarto & $5 \mathrm{cr}$. \\
\hline Universitat Oberta Catalunya (UOC) & $\begin{array}{l}\text { Ética y régimen jurídico de la publicidad y } \\
\text { las relaciones públicas }\end{array}$ & OP & $\begin{array}{l}\text { Segundo } \\
\text { ciclo }\end{array}$ & $6 \mathrm{cr}$. \\
\hline Universidad del País Vasco & Deontología de la Publicidad & OP & Tercero & $6 \mathrm{cr}$. \\
\hline Universitat Pompeu Fabra & Legislació i Deontologia Professional & OB & Cuarto & $3 \mathrm{cr}$. \\
\hline Universidad Pontificia de Salamanca & Ética y deontología de la comunicación & OB & Quinto & $7 \mathrm{cr}$. \\
\hline Universitat Ramon Llull & Ėtica de la Comunicació & OB & Cuarto & $5 \mathrm{cr}$. \\
\hline Universidad Rey Juan Carlos I & Ética y Deontología Profesional & OP & $\begin{array}{l}\text { Segundo } \\
\text { Ciclo }\end{array}$ & $6 \mathrm{cr}$. \\
\hline Universitat Rovira i Virgili (Tarragona) & $\begin{array}{l}\text { Deontologia Professional (Periodisme i } \\
\text { Publicitat) }\end{array}$ & OP & $\begin{array}{l}\text { Primer } \\
\text { ciclo }\end{array}$ & $4,5 \mathrm{cr}$. \\
\hline Universidad de Sevilla & Problemas éticos actuales de la publicidad & OP & Cuarto & $4,5 \mathrm{cr}$. \\
\hline Universidad de Valladolid & $\begin{array}{l}\text { Ética y Deontología de la Publicidad y de } \\
\text { las Relaciones Públicas }\end{array}$ & OB & Cuarto & $4 \mathrm{cr}$. \\
\hline Universitat de Vic & $x$ & $x$ & $x$ & $x$ \\
\hline \multirow[t]{2}{*}{ Universidad de Vigo } & Ética y Deontología & OB & Tercero & $6 \mathrm{cr}$. \\
\hline & $\begin{array}{l}\text { Práctica Y Deontología de la } \\
\text { Comunicación }\end{array}$ & OP & Tercero & $6 \mathrm{cr}$. \\
\hline
\end{tabular}

Fuente: Elaboración propia 


\section{El caso de la Universidad de Alicante}

La Deontología de la Publicidad y de las Relaciones Públicas, cuyo descriptor reza: “Bases éticas que deben regular la actividad publicitaria y las relaciones públicas, más allá del marco legal", se contempló como obligatoria ya en el primer Plan de Estudios de Publicidad y RR.PP. de la UA y empezó a impartirse en esta titulación en el curso 98/99. La materia cuenta con 4,5 créditos, de los cuales 3 son teóricos y 1,5 prácticos. La Deontología se concibe como una asignatura con un marcado carácter aplicado (en cuanto a autorregulación y profesionalismo se refiere) con una profunda conexión con todas aquellas asignaturas específicas de la profesión publicitaria que el alumno recibe a lo largo de la carrera. El programa se va impartiendo en clases teóricas (con ayuda de un manual-Martín Llaguno, 2006) y en clases prácticas, mediante el método del caso. Los objetivos específicos de esta asignatura, que comprenden distintas dimensiones, se presentan en la tabla siguiente (ver tabla 2):

Tabla 2. Objetivos específicos de la materia Deontología de la Publicidad y de las RR.PP. (UA)

\section{Dimensión cognitiva}

Reconocer los valores fundamentales de la publicidad en la sociedad actual y los derechos a los que satisface;

Considerar los derechos y responsabilidades morales que adquiere el publicitario;

Aprender las normas generales (legales y deontológicas) que deben presidir el comportamiento de un buen profesional

Identificar algunos de los problemas éticos a los que se va a enfrentar en el ejercicio de su profesión;

Conocer los principales sistemas de control que tiene la publicidad y su funcionamiento;

Familiarizarse con los principales códigos deontológicos de la profesión.

Dimensión técnica

Saber aplicar el saber genérico de la ética a las circunstancias específicas de la profesión;

Saber manejar algunos de los instrumentos básicos de la disciplina;

Perfeccionar la expresión oral y escrita.

Dimensión social

Desarrollar el sentido del compañerismo y la capacidad de relación;

Ser capaz de trabajar en equipo y de poner en práctica el liderazgo compartido;

Aumentar la capacidad de diálogo y de discusión y la empatía;

Desarrollar habilidades sociales y la capacidad de negociación.

Dimensión afectiva

Ser capaz de enfrentarse y resolver conflictos;

Ser capaz de evaluar hechos e ideas;

Desarrollar la autocrítica y la autoevaluación;

Desarrollar la identidad con la profesión

Incentivar la capacidad de superación, la autoestima y el pensamiento positivo. 


\section{Dimensión ética}

Despertar la sensibilidad moral y el interés por las cuestiones relacionadas con la deontología;

Fomentar el sentido de la profesionalidad, la honestidad, la responsabilidad y la justicia;

Transmitir al alumno en tanto que ciudadano y profesional el valor de la tolerancia, del pensamiento crítico y la responsabilidad;

Inculcar la sensibilidad social y la prudencia mostrándole las opiniones sobre las repercusiones que, el día de mañana, su trabajo puede tener sobre la sociedad;

Hacer al alumno capaz de tomar decisiones y de argumentarlas desde una perspectiva ética.

Fuente: Martín-Llaguno (2006)

Una de las cuestiones especialmente relevante en la materia de deontología es el sistema de evaluación. En efecto, dados los objetivos expuestos de la asignatura, al margen de las competencias cognitivas-técnicas (que se valoran de forma continuada a lo largo del curso a través de los ejercicios prácticos y coyunturalmente con un examen final), en ética profesional resulta trascendental servirse de un instrumento para controlar los cambios de actitudes y comportamientos de los alumnos (dimensiones afectivas, sociales y éticas).

\subsection{Objetivos}

En el contexto anterior, el objetivo concreto de este trabajo, enmarcado en un proyecto longitudinal sobre el efecto de la enseñanza de la ética, es evaluar el impacto de un curso de deontología publicitaria en las actitudes hacia las dimensiones profesionales de los estudiantes de comunicación comercial en España, con especial atención a las diferencias por sexo.

De manera más específica, nos planteamos:

a) Valorar los cambios en las actitudes de los estudiantes hacia las distintas dimensiones del profesionalismo después de un curso de deontología profesional;

b) Valorar el impacto del género en el cambio de estas actitudes, analizando si existen diferencias entre sexos en las puntuaciones concedidas a las distintas competencias.

\section{Metodología}

\subsection{Medidas}

Para el logro de nuestros objetivos (valorar las influencias actitudinales de la deontología) nos basamos en el instrumento Escala de Actitudes Éticas de Hirsch (2005) que ha sido validado en estudiantes universitarios españoles de otras titulaciones. Se trata de una escala que mide las actitudes hacia distintos aspectos de las competencias profesionales, 
compuesta por 55 ítems distribuidos en cuatro subescalas: (1) Actitud hacia competencias cognitivas; (2) Actitud hacia competencias sociales; (3) Actitud hacia competencias afectivoemocionales; (4) Actitud hacia competencias éticas. Tras realizar un análisis factorial de componentes principales con rotación varimax y siguiendo los análisis realizados por Hirsch (2005), obtuvimos cuatro dimensiones que transformamos en escalas. La tabla 3 muestra los ítems que componen cada una de las dimensiones con las puntuaciones medias globales para nuestra muestra y las Alfas de Chronbach para cada dimensión.

Tabla 3. Asertos en cada dimensión

\begin{tabular}{|c|c|c|}
\hline Actitud hacia Competencia Cognitiva $(a=0,81)$ & Media & $\begin{array}{l}\text { Desv. } \\
\text { típ. }\end{array}$ \\
\hline Me gusta tratar con profesionales que tengan buenos conocimientos científicos & 4,57 & 0,47 \\
\hline Me produce satisfacción la adquisición de nuevos conocimientos profesionales & 4,75 & 0,37 \\
\hline La puesta al día en los conocimientos es imprescindible para ser un buen profesional & 4,67 & 0,42 \\
\hline $\begin{array}{l}\text { Si no estoy preparándome continuamente no puedo resolver nuevas situaciones y } \\
\text { problemas profesionales }\end{array}$ & 4,02 & 0,77 \\
\hline $\begin{array}{l}\text { Para no cometer errores en mi ejercicio profesional debo ser consciente de los } \\
\text { límites de mis conocimientos y habilidades }\end{array}$ & 4,19 & 0,68 \\
\hline $\begin{array}{l}\text { Estoy dispuesto/a a ocupar tiempo en actualizar mis conocimientos sobre algún } \\
\text { aspecto de mi profesión }\end{array}$ & 4,37 & 0,53 \\
\hline Estoy dispuesto/a a dedicar dinero a mi formación & 4,25 & 0,61 \\
\hline Es necesario hacer cosas nuevas para avanzar en la profesión & 4,48 & 0,52 \\
\hline Disfruto cuando tengo que aprender algo nuevo & 4,28 & 0,61 \\
\hline \multicolumn{3}{|l|}{ Actitud hacia Competencia Social $(a=0,747)$} \\
\hline $\begin{array}{l}\text { Me siento mejor cuando tengo presente las necesidades profesionales de mis } \\
\text { compañeros }\end{array}$ & 3,96 & 0,58 \\
\hline Me gusta tratar con profesionales que toman en cuenta mi opinión & 4,81 & 0,31 \\
\hline $\begin{array}{l}\text { Me gusta que mis compañeros de trabajo valoren positivamente mi buen trato con } \\
\text { las personas }\end{array}$ & 4,45 & 0,53 \\
\hline Puedo resolver importantes cuestiones profesionales escuchando a los demás & 4,24 & 0,57 \\
\hline Actúo como un buen profesional cuando soy sensible a las necesidades de los demás & 4,18 & 0,61 \\
\hline Prefiero trabajar en equipo porque el resultado es de más alta calidad & 3,80 & 0,84 \\
\hline $\begin{array}{l}\text { Se equivocan las instituciones que no promueven el trabajo en equipo de sus } \\
\text { profesionales }\end{array}$ & 3,94 & 0,75 \\
\hline Trabajar con ahínco es parte de mi realización personal & 4,29 & 0,59 \\
\hline \multicolumn{3}{|l|}{ Actitud hacia Competencia Ética $(a=0,85)$} \\
\hline Estoy dispuesto/a a asumir las consecuencias de mis errores profesionales & 4,59 & 0,46 \\
\hline La confidencialidad es importante en el ejercicio profesional & 4,44 & 0,57 \\
\hline El cumplimiento a tiempo de mis compromisos profesionales es importante & 4,67 & 0,44 \\
\hline $\begin{array}{l}\text { Debo ganarme la confianza de las personas para las que trabajo actuando con } \\
\text { honestidad }\end{array}$ & 4,53 & 0,45 \\
\hline Hay decisiones éticas tan importantes en el ejercicio de mi profesión que no puedo & 4,08 & 0,56 \\
\hline
\end{tabular}




\begin{tabular}{|c|c|c|}
\hline dejarlas sólo a criterio de las organizaciones & & \\
\hline $\begin{array}{l}\text { Estoy convencido/a de que para ser un buen profesional tendré que hacer algún tipo } \\
\text { de sacrificio }\end{array}$ & 4,15 & 0,62 \\
\hline $\begin{array}{l}\text { La formación en ética puede ser necesaria para enfrentarme a conflictos en el } \\
\text { trabajo profesional }\end{array}$ & 4,41 & 0,53 \\
\hline $\begin{array}{l}\text { Considero imprescindible tener en cuenta los aspectos éticos en el ejercicio de mi } \\
\text { profesión }\end{array}$ & 4,47 & 0,51 \\
\hline $\begin{array}{l}\text { No debo tomar decisiones profesionales importantes sin antes valorar sus } \\
\text { consecuencias }\end{array}$ & 4,54 & 0,57 \\
\hline $\begin{array}{l}\text { Para mi buen ejercicio profesional no puedo limitarme a desarrollar sólo las } \\
\text { habilidades técnicas }\end{array}$ & 4,26 & 0,57 \\
\hline $\begin{array}{l}\text { Para ser un buen profesional no puedo ignorar los problemas de la sociedad en la } \\
\text { que vivo }\end{array}$ & 4,58 & 0,52 \\
\hline No estoy dispuesto/a a ejercer mi profesión sólo por dinero & 3,72 & 0,81 \\
\hline En la profesión, ayudar a los demás es más importante que alcanzar el éxito & 3,60 & 0,76 \\
\hline El éxito profesional no significa nada si no me permite ser una mejor persona & 3,93 & 0,73 \\
\hline De nada sirve un trabajo bien hecho si no contribuye a ayudar a los demás & 3,77 & 0,71 \\
\hline $\begin{array}{l}\text { Un buen profesional debe darse tiempo para evaluar las consecuencias de sus } \\
\text { acciones }\end{array}$ & 4,32 & 0,50 \\
\hline Seleccioné mi carrera para ser útil a las personas & 2,92 & 0,98 \\
\hline La coherencia con los principios éticos es más importante que ganar dinero & 3,89 & 0,77 \\
\hline Respeto las opiniones de los clientes y usuarios sobre mi trabajo profesional & 4,24 & 0,56 \\
\hline Transmito mis propios valores a través del ejercicio profesional & 4,14 & 0,64 \\
\hline Actitud hacia Competencia Afectiva $(a=0,713)$ & & \\
\hline Estoy satisfecho/a con la profesión que he elegido & 4,02 & 0,83 \\
\hline Es más fácil desarrollar el trabajo si se está identificado con la profesión & 4,29 & 0,58 \\
\hline La profesión es un ámbito de identidad para sus miembros & 3,97 & 0,64 \\
\hline $\begin{array}{l}\text { En el ejercicio profesional ayuda mucho tener una actitud positiva ante la adversidad } \\
\text { y la superación de retos }\end{array}$ & 4,49 & 0,51 \\
\hline Es bueno tener aspiraciones pero no una ambición desmedida & 1,64 & 0,76 \\
\hline $\begin{array}{l}\text { Considero conveniente aceptar el riesgo de equivocarme con tal de mejorar mi } \\
\text { actividad profesional }\end{array}$ & 4,14 & 0,59 \\
\hline $\begin{array}{l}\text { La solución de los problemas sociales es un asunto técnico que hace innecesario } \\
\text { escuchar a los ciudadanos }\end{array}$ & 1,91 & 1,02 \\
\hline Para tomar buenas decisiones profesionales hay que tener seguridad en uno mismo & 4,48 & 0,51 \\
\hline
\end{tabular}

Se obtuvo el Coeficiente Alpha de Chronbach (que mide la consistencia interna) con un resultado de 0,94 para la escala total.

\subsection{Muestra}

La Escala de Actitudes Éticas de Hirsch se administró en formato on-line, a través de la Intranet de la UA, a una muestra de 155 estudiantes -42 hombres $(27,1 \%)$ y 113 mujeres 
(72,9\%)- representativos para la licenciatura de Publicidad y Relaciones Públicas de la Universidad de Alicante (nivel de confianza = 95\%; precisión absoluta $=1,027 \%$; $p=0,5$ ), durante el curso 2008-2009 en dos períodos temporales:

a) De forma previa a la impartición de la materia deontología publicitaria.

b) De forma posterior a la impartición de dicha asignatura.

\section{Resultados}

4.1. Cambios en las actitudes de los estudiantes hacia las distintas dimensiones del profesionalismo después de un curso de deontología profesional

La Tabla 4 (ver tabla 4) presenta las puntuaciones medias de las actitudes de los alumnos hacia las competencias evaluadas, de manera general, en el tiempo 1 (antes del curso) y en el tiempo 2 (después del curso). Como se puede observar, en primer lugar, la actitud más favorable por parte de los alumnos es hacia las competencias cognitivas, seguidas de las competencias afectivas, sociales y éticas. En segundo lugar, todas las actitudes hacia las competencias mejoran después del curso. En tercer lugar, y dentro de esta tendencia, la sensibilidad hacia las competencias sociales experimenta la mayor diferencia relativa y gana un escalafón en el ranking de las dimensiones tras cursar la materia.

Tabla 4. Descriptivos de las actitudes globales, en el tiempo 1 y en el tiempo 2

\begin{tabular}{|l|l|l|l|l|l|l|l|}
\hline Variables & N & Media & $\begin{array}{l}\text { Desv. } \\
\text { típ. }\end{array}$ & Media & $\begin{array}{l}\text { Desv. } \\
\text { típ. }\end{array}$ & $\begin{array}{l}\text { Media } \\
\text { típ. }\end{array}$ \\
\hline Actitud hacia Competencia Cognitiva & 155 & 4,40 & 0,34 & 4,36 & 0,42 & 4,43 & 0,37 \\
\hline Actitud hacia Competencia Social & 155 & 4,21 & 0,34 & 4,16 & 0,42 & 4,26 & 0,38 \\
\hline Actitud hacia Competencia Ética & 155 & 4,16 & 0,36 & 4,13 & 0,42 & 4,19 & 0,38 \\
\hline Actitud hacia Competencia Afectiva & 155 & 4,20 & 0,33 & 4,17 & 0,40 & 4,23 & 0,37 \\
\hline
\end{tabular}

El aserto anterior es corroborado en la tabla 5 (ver tabla 5) donde, en la primera columna, se puede observar el diferencial en las medias de las puntuaciones de actitudes para cada competencia y, en la última columna, la significatividad estadística de estas diferencias en todos los casos. 
Tabla 5. Comparaciones de medias para muestras relacionadas de las actitudes en el tiempo 1 y en el tiempo 2

\begin{tabular}{|c|c|c|c|c|c|c|c|c|}
\hline \multirow[t]{3}{*}{ Variables } & \multicolumn{5}{|c|}{ Diferencias relacionadas } & \multirow[t]{3}{*}{$\mathbf{t}$} & \multirow[t]{3}{*}{ gl } & \multirow{3}{*}{$\begin{array}{l}\text { Sig. } \\
\text { bilate } \\
\text { ral }\end{array}$} \\
\hline & \multirow[t]{2}{*}{ Media } & \multirow[t]{2}{*}{$\begin{array}{l}\text { Desv. } \\
\text { típ. }\end{array}$} & \multirow{2}{*}{$\begin{array}{l}\text { Error } \\
\text { típico } \\
\text { de la } \\
\text { medía }\end{array}$} & \multicolumn{2}{|c|}{$\begin{array}{l}95 \% \text { Intervalo de } \\
\text { confianza para la } \\
\text { diferencia }\end{array}$} & & & \\
\hline & & & & Superior & Inferior & & & \\
\hline $\begin{array}{l}\text { Competencia Cognitiva1 } \\
\text { Competencia Cognitiva2 }\end{array}$ & $-0,07$ & 0,41 & 0,03 & $-0,14$ & $-0,01$ & $-2,24$ & 154 & 026 \\
\hline $\begin{array}{l}\text { Competencia Social1 } \\
\text { Competencia Social2 }\end{array}$ & $-0,10$ & 0,43 & 0,03 & $-0,16$ & $-0,03$ & $-2,78$ & 154 &, 006 \\
\hline $\begin{array}{l}\text { Competencia Ética1 } \\
\text { Competencia Ética2 }\end{array}$ & $-0,06$ & 0,37 & 0,03 & $-0,12$ & $-0,00$ & $-2,13$ & 154 & 035 \\
\hline $\begin{array}{l}\text { Competencia Afectiva1 } \\
\text { Competencia Afectiva2 }\end{array}$ & $-0,06$ & 0,38 & 0,03 & $-0,12$ & $-0,00$ & $-1,99$ & 154 & 049 \\
\hline
\end{tabular}

4.2. Cambios en las actitudes de los estudiantes hacia las distintas dimensiones del profesionalismo en función del sexo

La prueba T para muestras relacionadas, segmentando por sexo, indica, sin embargo, que estos cambios de actitud son significativos en los hombres, pero no entre las mujeres (ver tabla 6).

Tabla 1. Comparaciones de medias para muestras relacionadas de las actitudes en el tiempo 1 y en el tiempo 2, en el grupo de hombres y de mujeres

\begin{tabular}{|c|c|c|c|c|c|c|c|c|c|}
\hline \multirow[t]{3}{*}{ Sexo } & \multirow[t]{3}{*}{ Variables } & \multicolumn{5}{|c|}{ Diferencias relacionadas } & \multirow[t]{3}{*}{$\mathbf{t}$} & \multirow[t]{3}{*}{ gl } & \multirow{3}{*}{$\begin{array}{l}\text { Sig. } \\
\text { bilater } \\
\text { al }\end{array}$} \\
\hline & & \multirow[t]{2}{*}{ Media } & \multirow[t]{2}{*}{$\begin{array}{l}\text { Desv. } \\
\text { típ. }\end{array}$} & \multirow{2}{*}{$\begin{array}{l}\text { Error } \\
\text { típico } \\
\text { de la } \\
\text { media }\end{array}$} & \multicolumn{2}{|c|}{$\begin{array}{l}95 \% \text { Intervalo de } \\
\text { confianza para la } \\
\text { diferencia }\end{array}$} & & & \\
\hline & & & & & Superior & Inferior & & & \\
\hline Hombre & $\begin{array}{l}\text { Competencia } \\
\text { Cognitiva1 } \\
\text { Competencia } \\
\text { Cognitiva2 }\end{array}$ & $-0,17$ & 0,48 & 0,07 & $-0,32$ & $-0,02$ & $-2,23$ & 41 & 031 \\
\hline Hombre & $\begin{array}{l}\text { Competencia } \\
\text { Social1 } \\
\text { Competencia } \\
\text { Social2 }\end{array}$ & $-0,18$ & 0,48 & 0,07 & $-0,33$ & $-0,03$ & $-2,39$ & 41 & ,022 \\
\hline Hombre & $\begin{array}{l}\text { Competencia } \\
\text { Étical } \\
\text { Competencia } \\
\text { Ética2 }\end{array}$ & $-0,16$ & 0,48 & 0,08 & $-0,31$ & $-0,01$ & $-2,14$ & 41 & 038 \\
\hline
\end{tabular}




\begin{tabular}{|l|l|l|l|l|l|l|l|l|l|}
\hline Hombre & $\begin{array}{l}\text { Competencia } \\
\text { Afectiva1 } \\
\text { Competencia } \\
\text { Afectiva2 }\end{array}$ & $-0,15$ & 0,39 & 0,06 & $-0,27$ & $-0,03$ & $-2,56$ & 41 &, 014 \\
\hline Mujer & $\begin{array}{l}\text { Competencia } \\
\text { Cognitiva1 } \\
\text { Competencia } \\
\text { Cognitiva2 }\end{array}$ & $-0,04$ & 0,38 & 0,04 & $-0,11$ & 0,03 & $-1,11$ & 112 &, 268 \\
\hline Mujer & $\begin{array}{l}\text { Competencia } \\
\text { Social1 } \\
\text { Competencia } \\
\text { Social2 }\end{array}$ & $-0,06$ & 0,40 & 0,04 & $-0,14$ & 0,01 & $-1,71$ & 112 &, 091 \\
\hline Mujer & $\begin{array}{l}\text { Competencia } \\
\text { Ética1 } \\
\text { Competencia } \\
\text { Ética2 }\end{array}$ & $-0,03$ & 0,31 & 0,03 & $-0,09$ & 0,03 & $-0,93$ & 112 &, 352 \\
\hline $\begin{array}{l}\text { Competencia } \\
\text { Afectiva1 } \\
\text { Competencia } \\
\text { Afectiva2 }\end{array}$ & $-0,03$ & 0,38 & 0,04 & $-0,10$ & 0,04 & $-0,77$ & 112 &, 444 \\
\hline
\end{tabular}

Como muestra la tabla 7 (ver tabla 7), tanto hombres como mujeres siguieron la misma pauta: experimentaron cambios en todas y cada una de las dimensiones, con especial relevancia en el caso de la competencia social y en el caso de los hombres.

Las mujeres puntúan más alto que los hombres en todas las competencias profesionales (ver medias de las COMPETENCIAS en la tabla 7), pero los cambios de actitudes han sido mayores en general en los hombres (ver medias de los DIFERENCIALES en la tabla 7). Sin embargo, la prueba de comparación de medias para muestras independientes con el diferencial entre el tiempo dos y el tiempo uno no arroja significación estadística entre ambos sexos. Es decir, aunque el curso de deontología consiguió incrementar más la sensibilidad ética en varones que en mujeres, este incremento de la sensibilidad en los varones no fue lo suficientemente grande como para arrojar significatividad estadística.

Tabla 2. Descriptivos de las competencias globales y las diferencias en las actitudes en el tiempo 1 y 2 en función de sexo

\begin{tabular}{|l|l|l|l|l|}
\hline Variable & Sexo & Media & Desv. Típ. & F; sig \\
\hline \multirow{2}{*}{ Competencia Cognitiva global } & Hombre & 4,24 & 0,42 & \\
\cline { 2 - 4 } & Mujer & 4,46 & 0,29 & \\
\hline \multirow{2}{*}{ DIFERENCIAL COGNITIVA2- COGNITIVA1 } & Hombre & 0,17 & 0,48 & 0,$44 ;$ \\
\cline { 2 - 4 } & Mujer & 0,04 & 0,38 & 0,086 \\
\hline \multirow{2}{*}{ Competencia Social Global } & Hombre & 4,01 & 0,36 & \\
\cline { 2 - 5 } & Mujer & 4,28 & 0,31 & \\
\hline \multirow{2}{*}{ DIFERENCIAL SOCIAL2-SOCIAL1 } & Hombre & 0,18 & 0,48 & 0,$31 ;$ \\
\cline { 2 - 5 } & Mujer & 0,06 & 0,40 & 0,13 \\
\hline
\end{tabular}




\begin{tabular}{|l|l|l|l|l|}
\hline \multirow{2}{*}{ Competencia Ética Global } & Hombre & 3,97 & 0,35 & \\
\cline { 2 - 4 } & Mujer & 4,23 & 0,33 & \\
\hline \multirow{2}{*}{ DIFERENCIAL ÉTICA2-ÉTICA1 } & Hombre & 0,16 & 0,48 & 5,$9 ;$ \\
& Mujer & 0,03 & 0,31 & \\
\hline \multirow{2}{*}{ Competencia Afectiva Global } & Hombre & 3,90 & 0,37 & \\
\cline { 2 - 5 } & Mujer & 4,01 & 0,30 & 4,$1 ;$ \\
\hline \multirow{2}{*}{ DIFERENCIAL AFECTIVA2-AFECTIVA1 } & Hombre & 0,07 & 0,45 & 0,7 \\
\hline & Mujer & 0,04 & 0,38 & \multirow{2}{*}{} \\
\hline
\end{tabular}

\section{Discusión}

Los imperativos de la convergencia europea están suponiendo importantes cambios para la docencia, cuyo objetivo general es ahora el "aprendizaje autónomo" del alumno, y que ha de ser planificada por "competencias profesionales" y teniendo presente la idea del "Ionglife learning" o la enseñanza continua. En este proceso se hace necesario también adaptar los métodos de evaluación. Evaluar es asignar un valor a algo. Valorar la dimensión actitudinal y comportamental en el caso de la deontología nos parece una actividad tan importante, o más, que la evaluación de la dimensión cognitiva. Si bien valorar la dimensión comportamental del aprendizaje a través de la materia exige el diseño de proyectos a largo plazo que monitoreen a los egresados, la escala de Actitudes Éticas de Hirsh se muestra como un instrumento válido para medir los cambios de actitudes.

Así, la deontología profesional incide en la disposición de los estudiantes hacia las cuatro dimensiones del profesionalismo. Los resultados de nuestro trabajo apuntan, en primer lugar, que, tal y como se había previsto, al margen de la repercusión sobre los conocimientos - que se valora con los exámenes- el curso de deontología de la publicidad de la UA tiene efectos sobre las actitudes. En este sentido, la inclusión de este tipo de materia en la curricula parece ser eficaz en el incremento de la sensibilidad hacia el profesionalismo en general y hacia la ética en concreto. En la línea de lo apuntado por Surlin (1987), Gale y Bunton (2005) y Stead y Miller (1988), los alumnos que han cursado la materia presentan cambios en el sistema de valores - especialmente relacionados con la dimensión social- y un aumento de la importancia concedida a las cuestiones profesionales y morales.

El especial incremento de la actitud favorable hacia la dimensión social que las clases de deontología consiguen no es baladí. En una profesión con un marcado carácter relacional, saber trabajar en equipo y saber relacionarse con los compañeros de trabajo son, entre otras, algunas de las habilidades que obtienen una mejor puntuación tras un cuatrimestre de formación en cuestiones éticas. No en vano, la identificación mental y afectiva con los problemas de otros sujetos (la empatía) es una de las aproximaciones utilizadas para la resolución de los dilemas éticos. Además, fomentar la "creatividad moral" y saber adoptar 
por consenso una solución a un conflicto forman parte de las competencias sociales desarrolladas en la asignatura.

Por otra parte, y como planteaban otros trabajos (Becker y Ulstad, 2007; Ruegger y King, 1992; Betz, 1989), las mujeres puntúan más alto que los hombres en las actitudes hacia todas las competencias profesionales desde el inicio. Sin embargo, la incidencia del curso de deontología es ligeramente mayor para los chicos que para las chicas. En consonancia con la hipótesis del knowledge gap (Tichenor, Donohue y Olien, 1970), el attitude gap podría dar cuenta de que en aquellos sujetos más sensibles hacia una dimensión, la modificación de las actitudes que se consigue con una intervención es menor. En una profesión crecientemente feminizada como la publicitaria (Martín-Llaguno, Beléndez y Hernández, 2008) la especial predisposición de la mujer hacia las cuestiones éticas podría tener repercusiones importantes sobre los procesos y los productos de la industria.

Para finalizar, este trabajo no permite aventurar a) en qué medida los efectos de la enseñanza de la deontología son a corto, a medio, o a largo plazo y b) si nuestro esfuerzo consigue incidir en el proceso final y traspasar de las actitudes a los comportamientos. Para poder valorar ambos aspectos se hace necesaria la realización de estudios longitudinales que analicen si existe una correspondencia entre la conformación de las actitudes éticas, durante la etapa universitaria, y su manifestación efectiva, en forma de comportamiento, durante el ejercicio profesional. Sólo de esta manera podremos conocer de forma fehaciente el calado de la enseñanza de la ética profesional en los profesionales de la publicidad.

En esta línea, nos parece relevante observar en futuros estudios la incidencia de la experiencia profesional previa sobre las actitudes éticas de los alumnos. De este modo, se podría valorar el peso de la academia y la profesión en la aculturación profesional de los estudiantes. Además para comprender parte de la idiosincrasia de la profesión del publicitario, sería necesario contrastar los resultados de este trabajo con los obtenidos por los pre-profesionales de otras titulaciones.

\section{REFERENCIAS BIBLIOGRÁFICAS}

BARNEY, R. D. y BLACK, J. (1994): "Ethics and Professional Persuasive Communications", en Public Relations Review, vol. 20, no3, pp. 233-49.

BECKER, D. A. y ULSTAD, I. (2007): "Gender Differences in Student Ethics: Are Females Really More Ethical?", en Plagiary: Cross-Disciplinary Studies in Plagiarism, Fabrication, and Falsification, vol. 2, no 3, pp. 1-15.

BETZ, Michael (1989): "Gender Differences in Proclivity for Unethical Behavior", en J ournal of Business Ethics, vol. 8, no 5, pp.321-324.

BIVINS, T. H. (1990): "Are Public Relations Texts Covering Ethics Adequately?", en J ournal of Mass Media Ethics, vol.4, no1, pp. 39-52. 
BORKOWSKI, Susan C. y UGRAS, Yusuf J. (1992): "The Ethical Attitudes of Students as a Function of Age, Sex and Experience", en Journal of Business Ethics, vol. 11, no 12, pp. 961-979.

BRAUN, M. J. (1999): "Media Ethics Education: A Comparison of Student Responses", en Journal of Mass Media Ethics, vol.14, no3, pp.171-82.

CARLSON, P. J. y BURKE, F. (1998): "Lessons Learned from Ethics in the Classroom", en Journal of Business Ethics, vol.17, no 11, pp. 1179-1187.

COSTA, Joan (2004): La imagen de marca, un fenómeno social. Barcelona, Paidós.

GALE, K. y BUNTON, K. (2005): "Assessing the Impact of Ethics Instruction on Advertising and Public Relation Graduates", en Journalism \& Mass Communication Educator, vol.60, no 3, pp. 272-285.

GAUTSCHI III, F. H., y JONES, T. M. (1998): "Enhancing the Ability of Business Students to Recognize Ethical Issues: An Empirical Assessment of the Effectiveness of a Course in Business Ethics," en Journal of Business Ethics, vol.17, no 2, pp. 205-216.

HARRISON, S. L. (1990): "Pedagogical Ethics for Public Relations and Advertising", en Journal of Mass Media Ethics, vol.5, no4, pp- 256- 262.

HIRSCH, Ana (2005): "Construcción de una escala de actitudes sobre ética profesional", en Revista Electrónica de Investigación Educativa, vol.7, $\mathrm{n}$ 느, en http://redie.uabc.mx/vol7nol/contenido-hirsch.html (consultado 13/11/2007).

JIANAKOPOLOS, N.A. y BERNASEK, A. (1998): "Are women more risk averse?", en Economic Inquiry, vol. 36, no 4, pp. 620-630.

LÓPEZ LITA, R. y BERNAD, E. (2007): "Publicidad, medios de comunicación y segregación ocupacional de la mujer: perpetuación y superación de estereotipos de género y sus consecuencias en el mercado de mano de obra", en Revista del Ministerio de Trabajo e Inmigración, no 67, pp. 213-226.

MARTín-LLAGUNO, Marta (2006): Proyecto docente y programa razonado. Alicante, Limencop.

MARTín-LLAGUNO, M., BELÉNDEZ, M. y HERNÁNDEZ, A. (2008): La mujer en las agencias de publicidad. Categorías, especializaciones y conflicto trabajo-familia en las agencias españolas. Madrid, AEAP. Disponible en http://www.aeap.es/publicaciones/docs /LaMujerSectorPublicitario.pdf (consultado 23/05/2009).

MARTÍn-LLAGUNO, M. y HERNÁNDEZ, A. (2009): “El control de la comunicación comercial en un mundo globalizado. Regulación, autorregulación e hiperregulación de la publicidad", en El Portal de la Comunicación, en http://www.portalcomunicacion .com/esp/pdf/aab_lec/50.pdf (consultado: 12/08/2009)

MARTIN, T.R. (1981): "Do Courses in Ethics Improve the Ethical Judgment of Students?", en Business and Society, vol. 20, no2, pp.17-26.

MCBRIDE, G. (1989): "Ethical Thought in Public Relations History: Seeking a Relevant Perspective", en Journal of Mass Media Ethics, vol.4, no1, pp. 5-20.

MCCABE, A.C., INGRAM, R. y DATO-ON, M. C. (2006): "The business of ethics and gender", en J ournal of Business Ethics, vol. 64, nำ2, pp. 101-116. 
PEPPAS, S. C. y DISKIN, B. A. (2001): "College Courses in Ethics: Do They Really Make a Difference?", en The International Journal of Educational Management, vol.15, no, pp. 347-353.

PÉREZ PULIDO, Margarita (2004): "La enseñanza de la ética y deontología de la información en los estudios universitarios de biblioteconomía y documentación", en BiD: Textos universitaris de biblioteconomia i documentación, no 13, en http://www2.ub.es /bid/consulta_articulos.php?fichero=13pulid2.htm (consultado: 15/02/2010).

PLUMLEY, J. y FERRAGINA, Y. (1990): "Do Advertising Texts Cover Ethics Adequately?", en Journal of Mass Media Ethics, vol.5, no4, pp. 247-255.

RUEGGER, D. y KING, E. W. (1992): "A Study of the Effect of Age and Gender upon Student Business Ethics, en Journal of Business Ethics, vol. 11, no3, pp. 179-186.

SHANNON, J. R. y BERL, R. L. (1997): "Are We Teaching Ethics in Marketing? A Survey of Students' Attitudes and Perceptions", en Journal of Business Ethics, vol. 16, no10, pp. 1059-1075.

STEAD, B. A. y MILLER, J.J. (1988): "Can Social Awareness Be Increased Through Business School Curricula?", en Journal of Business Ethics, vol. 7, noㅜ, pp. 553-560.

SURLIN, S. H. (1987): "Value System Changes by Students as Result of Media Ethics Course", en Journalism Quarterly, vol. 64, pp.564-568.

TIBBETTS, S.G. (1997): "Gender differences in students' rational decisions to cheat", en Deviant Behavior, vol. 18, no 4, pp. 393-414.

TIBBETTS, S.G. (1999): "Differences between women and men regarding decisions to commit test cheating", en Research in Higher Education, vol. 40, no 3, pp. 323-342.

TICHENOR, P.J., DONOHUE, G.A. y OLIEN, C.N. (1970): "Mass Media Flow and Differential Growth in Knowledge", en Public Opinion Quarterly, vol. 34, pp. 159-170.

TUCKER, E. M. y STOUT, D. A. (1999): "Teaching Ethics: The Moral Development of Educators", en Journal of Mass Media Ethics, vol.14, no, pp. 107-118.

WRIGHT, D. K. (1985): "Age and the Moral Values of Practitioners", en Public Relations Review, vol. 11, no 1 , pp. 51-60.

[Recibido: 7 de julio de 2009. Comunicación de necesidad de corrección: 3 de febrero de 2010. Aceptación de la versión modificada: 22 de abril de 2010.] 